\title{
On Poisson's Theorem of Building First Integrals for Ordinary Differential Systems
}

\section{A. F. Pranevich}

We consider Hamiltonian systems with $n$ degrees of freedom. Among the general methods of integration of Hamiltonian systems, the Poisson method is of particular importance. It allows one to find the additional (third) first integral of the Hamiltonian system by two known first integrals of the Hamiltonian system. In this paper, the Poisson method of building first integrals of Hamiltonian systems by integral manifolds and partial integrals is developed. Also, the generalization of the Poisson method for general ordinary differential systems is obtained.

Keywords: Hamiltonian system, Poisson's theorem, first integral, integral manifold, partial integral, Poisson bracket

\section{Introduction}

Consider a canonical Hamiltonian system with $n$ degrees of freedom

$$
\frac{d q_{i}}{d t}=\partial_{p_{i}} H(q, p), \quad \frac{d p_{i}}{d t}=-\partial_{q_{i}} H(q, p), \quad i=1, \ldots, n,
$$

where $q=\left(q_{1}, \ldots, q_{n}\right) \in \mathbb{R}^{n}$ and $p=\left(p_{1}, \ldots, p_{n}\right) \in \mathbb{R}^{n}$ are the generalized coordinates and momenta, respectively, $t \in \mathbb{R}$, and the Hamiltonian $H$ is a twice continuously differentiable function on the domain $D \subset \mathbb{R}^{2 n}$.

Among the general methods of integration of the Hamiltonian system (1.1), the Poisson method is of particular importance. It makes it possible to find the additional (third) first integral of the Hamiltonian system (1.1) by two known first integrals of the Hamiltonian system (1.1). And thus, in certain cases, to build an integral basis of the Hamiltonian system (1.1). Due to this property, the Poisson method is included in almost all monographs and textbooks on analytical mechanics (see, for example, [1, pp. 298-306], [2, p. 216], and [3, pp. 85-86]) and is formulated as the following statement (the Poisson theorem).

Received August 21, 2018

Accepted March 19, 2019

Andrey F. Pranevich pranevich@grsu.by

Yanka Kupala State University of Grodno

ul. Ozechko 22, Grodno, 230023 Belarus 
Theorem 1. Suppose twice continuously differentiable functions $\mathrm{g}_{1}$ and $\mathrm{g}_{2}$ are first integrals on the domain $D$ of the Hamiltonian system (1.1). Then the Poisson bracket

$$
\mathrm{g}_{12}(q, p)=\left[\mathrm{g}_{1}(q, p), \mathrm{g}_{2}(q, p)\right] \text { for all }(q, p) \in D
$$

of the functions $\mathrm{g}_{1}$ and $\mathrm{g}_{2}$ is also a first integral of the Hamiltonian system (1.1).

In his Lectures on Dynamics [1, p. 303], C. G. J. Jacobi referred to Poisson's theorem as "one of the most remarkable theorems of the whole of integral calculus. In the particular case when $H=T-U$, it is the fundamental theorem of analytical mechanics."

Of course, Poisson's theorem does not always supply further first integrals. In some cases the result is trivial, the Poisson bracket being a constant. In other cases the first integral obtained is simply a function of the original integrals. If neither of these two possibilities occurs, however, then the Poisson bracket is a further first integral of the Hamiltonian system (1.1).

Our aim in this paper is to develop the Poisson method for integral manifolds and partial integrals (or Darboux polynomials) of the Hamiltonian system (1.1). Also, we focus our attention on generalizations of the Poisson method for general ordinary differential systems.

To avoid ambiguity, we stipulate the following notation and definitions. At the same time, we will mainly follow the approaches described in the monograph [4].

The Poisson bracket of functions $u, v \in C^{1}(D)$ is the function

$$
[u(q, p), v(q, p)]=\sum_{i=1}^{n}\left(\partial_{q_{i}} u(q, p) \partial_{p_{i}} v(q, p)-\partial_{p_{i}} u(q, p) \partial_{q_{i}} v(q, p)\right) \text { for all }(q, p) \in D .
$$

The functions $u$ and $v$ are in involution if $[u(q, p), v(q, p)]=0$ for all $(q, p) \in D$.

A function $\mathrm{g} \in C^{1}(D)$ is called a first integral on the domain $D$ of the Hamiltonian system $(1.1)$ if $\mathfrak{G} g(q, p)=0$ for all $(q, p) \in D$, where the linear differential operator

$$
\mathfrak{G}(q, p)=\sum_{i=1}^{n}\left(\partial_{p_{i}} H(q, p) \partial_{q_{i}}-\partial_{q_{i}} H(q, p) \partial_{p_{i}}\right) \text { for all }(q, p) \in D
$$

Notice that the Hamiltonian $H$ is a first integral on the domain $D$ of system (1.1).

A smooth manifold $\mathrm{g}(q, p)=0$ is said to be an integral manifold of the Hamiltonian system (1.1) if the derivative of the function $\mathrm{g} \in C^{1}(D)$ by virtue of the Hamiltonian system (1.1) is identically zero on the manifold $\mathrm{g}(q, p)=0$, i.e.,

$$
\mathfrak{G} \mathrm{g}(q, p)=\Phi(q, p), \quad \Phi(q, p)_{\left.\right|_{\mathrm{g}(q, p)=0}}=0 \text { for all }(q, p) \in D .
$$

If the Hamiltonian system (1.1) is polynomial, i.e., the Hamiltonian $H$ is a polynomial of degree $h$ in the variables $q$ and $p$, then we will use the notion of partial integral. A function $\mathrm{g} \in C^{1}(D)$ is called a partial integral on the domain $D$ of the polynomial Hamiltonian system (1.1) if the derivative of function $g$ by virtue of system (1.1) equals

$$
\mathfrak{G} \mathrm{g}(q, p)=\mathrm{g}(q, p) M(q, p) \text { for all }(q, p) \in D,
$$

where the polynomial $M$ in the variables $p$ and $q$ has the degree $\operatorname{deg}_{(p, q)} M \leqslant h-2$. Moreover, the polynomial $M$ is the cofactor of the partial integral $\mathrm{g}$.

The theory of partial integrals (or the Darboux theory of integrability) was established by the French mathematician Jean-Gaston Darboux [5] in 1878, which provided a link between the 
existence of first integrals and invariant algebraic curves (partial integrals) for polynomial autonomous differential systems. This theory is related to the Poincaré problem [6], which asks to find the upper bound of invariant algebraic curves of planar polynomial differential systems. The Darboux theory of integrability is also involved in the study of Hilbert's 16th problem (see, for example, [7]). For the current status of the theory of integrability see the monographs [4, 8-13] and references therein. Note also that recently the theory of partial integrals has been successfully applied to the study of some physical models (see, for instance, [14-19]).

Let $\mathrm{I}(\mathfrak{G})(\mathrm{M}(\mathfrak{G})$ and $\mathrm{J}(\mathfrak{G}))$ denote the set of all first integrals (integral manifolds and partial integrals) on the domain $D$ of system (1.1). The phrase "the function g defines an integral manifold of the Hamiltonian system (1.1) such that the identity (1.3) holds" is denoted by $(\mathrm{g}, \Phi) \in \mathrm{M}(\mathfrak{G})$. And the phrase "the function $\mathrm{g}$ is a partial integral with cofactor $M$ on the domain $D$ of the Hamiltonian polynomial system (1.1)" is denoted by $(\mathrm{g}, M) \in \mathrm{J}(\mathfrak{G})$.

The paper is organized as follows. In Section 2, we provide the Poisson method of building first integrals by known integral manifolds for the Hamiltonian differential system (1.1). In Section 3, we work with the notion of partial integral and state our main results about integral characteristics of the polynomial Hamiltonian differential system (1.1), which are determined by the Poisson bracket (1.2). Finally, in Section 4, we apply the results of Sections 2 and 3 to general ordinary differential systems.

\section{Integral manifolds}

Theorem 2. Suppose $\left(\mathrm{g}_{k}, \Phi_{k}\right) \in \mathrm{M}(\mathfrak{G})$ and $\mathrm{g}_{k} \in C^{2}(D), k=1,2$. Then the Poisson bracket $\left[\mathrm{g}_{1}, \mathrm{~g}_{2}\right] \in \mathrm{I}(\mathfrak{G})$ if and only if the following identity holds:

$$
\left[\mathrm{g}_{1}(q, p), \Phi_{2}(q, p)\right]=\left[\mathrm{g}_{2}(q, p), \Phi_{1}(q, p)\right] \text { for all }(q, p) \in D \text {. }
$$

Proof. Since $\left(\mathrm{g}_{k}, \Phi_{k}\right) \in \mathrm{M}(\mathfrak{G}), k=1,2$, we have (by the definition of integral manifold)

$$
\left[\mathrm{g}_{k}(q, p), H(q, p)\right]=\Phi_{k}(q, p) \quad \text { for all }(q, p) \in D, \quad k=1,2,
$$

where the functions $\Phi_{k}$ are such that $\left.\Phi_{k}(q, p)\right|_{\mathrm{g}_{k}(q, p)=0}=0$ for all $(q, p) \in D, k=1,2$.

Using these identities and the properties of Poisson brackets (Jacobi identity and anticommutativity), we obtain the derivative of the function (1.2) by virtue of system (1.1)

$$
\begin{gathered}
\mathfrak{G}\left[\mathrm{g}_{1}(q, p), \mathrm{g}_{2}(q, p)\right]=\left[\left[\mathrm{g}_{1}(q, p), \mathrm{g}_{2}(q, p)\right], H(q, p)\right]= \\
=\left[\left[\mathrm{g}_{1}(q, p), \mathrm{g}_{2}(q, p)\right], H(q, p)\right]+\left[\left[H(q, p), \mathrm{g}_{1}(q, p)\right], \mathrm{g}_{2}(q, p)\right]+\left[\left[\mathrm{g}_{2}(q, p), H(q, p)\right], \mathrm{g}_{1}(q, p)\right]- \\
-\left(\left[\left[H(q, p), \mathrm{g}_{1}(q, p)\right], \mathrm{g}_{2}(q, p)\right]+\left[\left[\mathrm{g}_{2}(q, p), H(q, p)\right], \mathrm{g}_{1}(q, p)\right]\right)=\left[\mathrm{g}_{1}(q, p),\left[\mathrm{g}_{2}(q, p), H(q, p)\right]\right]- \\
-\left[\mathrm{g}_{2}(q, p),\left[\mathrm{g}_{1}(q, p), H(q, p)\right]\right]=\left[\mathrm{g}_{1}(q, p), \Phi_{2}(q, p)\right]-\left[\mathrm{g}_{2}(q, p), \Phi_{1}(q, p)\right] \quad \text { for all }(q, p) \in D .
\end{gathered}
$$

Therefore, the Poisson bracket (1.2) of the integral manifolds $g_{1}$ and $g_{2}$ of system (1.1) is a first integral of the Hamiltonian system (1.1) if and only if the identity (2.1) is true.

Note that, under the conditions of Theorem 2, we get the following statement. If the function

$$
\Phi(q, p)=\left[\mathrm{g}_{1}(q, p), \Phi_{2}(q, p)\right]-\left[\mathrm{g}_{2}(q, p), \Phi_{1}(q, p)\right] \text { for all }(q, p) \in D
$$


such that the following identity holds:

$$
\left.\Phi(q, p)\right|_{\left[\mathrm{g}_{1}(q, p), \mathrm{g}_{2}(q, p)\right]=0}=0 \text { for all }(q, p) \in D,
$$

then the Poisson bracket (1.2) is an integral manifold of the Hamiltonian system (1.1).

As a consequence of Theorem 2, we obtain

Corollary 1. Let $\mathrm{g}_{1} \in \mathrm{I}(\mathfrak{G}),\left(\mathrm{g}_{2}, \Phi_{2}\right) \in \mathrm{M}(\mathfrak{G}), \mathrm{g}_{k} \in C^{2}(D), k=1,2$. Then the Poisson bracket $\left[\mathrm{g}_{1}, \mathrm{~g}_{2}\right] \in \mathrm{I}(\mathfrak{G})$ if and only if the functions $\mathrm{g}_{1}$ and $\Phi_{2}$ are in involution, i.e.,

$$
\left[\mathrm{g}_{1}(q, p), \Phi_{2}(q, p)\right]=0 \text { for all }(q, p) \in D \text {. }
$$

If $g_{1}, g_{2} \in \mathrm{I}(\mathfrak{G})$, then from Theorem 2 (or Corollary 1 ) we have the statement of the Poisson theorem (Theorem 1).

\section{Partial integrals}

In this section, we assume that the Hamiltonian system (1.1) is polynomial.

Proposition 1. Suppose $\left(\mathrm{g}_{k}, M_{k}\right) \in \mathrm{J}(\mathfrak{G})$ and $\mathrm{g}_{k} \in C^{2}(D), k=1,2$. Then the Poisson bracket $\left(\left[\mathrm{g}_{1}, \mathrm{~g}_{2}\right], M_{1}+M_{2}\right) \in \mathrm{J}(\mathfrak{G})$ if and only if the following identity holds

$$
\mathrm{g}_{2}(q, p)\left[\mathrm{g}_{1}(q, p), M_{2}(q, p)\right]-\mathrm{g}_{1}(q, p)\left[\mathrm{g}_{2}(q, p), M_{1}(q, p)\right]=0 \quad \text { for all }(q, p) \in D .
$$

Proof. Using a similar method as in the proof of Theorem 2, we obtain the following identity:

$$
\begin{gathered}
\mathfrak{G}\left[\mathrm{g}_{1}(q, p), \mathrm{g}_{2}(q, p)\right]=\left[\mathrm{g}_{1}(q, p), \mathrm{g}_{2}(q, p) M_{2}(q, p)\right]- \\
-\left[\mathrm{g}_{2}(q, p), \mathrm{g}_{1}(q, p) M_{1}(q, p)\right] \text { for all }(q, p) \in D .
\end{gathered}
$$

From the properties of Poisson brackets (Leibniz's rule and anticommutativity), we have

$$
\begin{gathered}
\mathfrak{G}\left[\mathrm{g}_{1}(q, p), \mathrm{g}_{2}(q, p)\right]=\left(M_{1}(q, p)+M_{2}(q, p)\right)\left[\mathrm{g}_{1}(q, p), \mathrm{g}_{2}(q, p)\right]+ \\
+\mathrm{g}_{2}(q, p)\left[\mathrm{g}_{1}(q, p), M_{2}(q, p)\right]-\mathrm{g}_{1}(q, p)\left[\mathrm{g}_{2}(q, p), M_{1}(q, p)\right] \quad \text { for all }(q, p) \in D
\end{gathered}
$$

Thus, the Poisson bracket (1.2) is a partial integral with cofactor $M_{1}+M_{2}$ of the Hamiltonian system (1.1) if and only if the identity (3.1) holds.

Taking into account the proof of Proposition 1, we get the following statement.

Theorem 3. Let $\left(\mathrm{g}_{k}, M_{k}\right) \in \mathrm{J}(\mathfrak{G}), \mathrm{g}_{k} \in C^{2}(D), k=1,2$. Then the Poisson bracket (1.2) is a first integral of the Hamiltonian system (1.1) if and only if this Poisson bracket on the domain $D_{0} \subset\left\{(q, p): M_{1}(q, p)+M_{2}(q, p) \neq 0\right\}$ has the form

$$
\left[\mathrm{g}_{1}(q, p), \mathrm{g}_{2}(q, p)\right]=\frac{\mathrm{g}_{1}(q, p)\left[\mathrm{g}_{2}(q, p), M_{1}(q, p)\right]-\mathrm{g}_{2}(q, p)\left[\mathrm{g}_{1}(q, p), M_{2}(q, p)\right]}{M_{1}(q, p)+M_{2}(q, p)} .
$$

Theorem 4. Suppose the Hamiltonian system (1.1) has partial integrals $\left(\mathrm{g}_{k}, M_{k}\right) \in \mathrm{J}(\mathfrak{G})$, $\mathrm{g}_{k} \in C^{2}(D), k=1,2$, such that the sum of cofactors

$$
M_{1}(q, p)+M_{2}(q, p)=0 \text { for all }(q, p) \in D,
$$


and the following identity holds:

$$
\mathrm{g}_{2}(q, p)\left[\mathrm{g}_{1}(q, p), M_{1}(q, p)\right]+\mathrm{g}_{1}(q, p)\left[\mathrm{g}_{2}(q, p), M_{1}(q, p)\right]=0 \quad \text { for all }(q, p) \in D .
$$

Then the Poisson bracket (1.2) is a first integral of the Hamiltonian system (1.1).

Proof. From Proposition 1 it follows that if the identities (3.3) and (3.4) are true, then the Poisson bracket (1.2) is a first integral of the Hamiltonian system (1.1).

Proposition 2. Suppose $\mathrm{g}_{1} \in \mathrm{I}(\mathfrak{G}),\left(\mathrm{g}_{2}, M_{2}\right) \in \mathrm{J}(\mathfrak{G})$, and $\mathrm{g}_{k} \in C^{2}(D), k=1,2$. Then the Poisson bracket $\left(\left[\mathrm{g}_{1}, \mathrm{~g}_{2}\right], M_{2}\right) \in \mathrm{J}(\mathfrak{G})$ if and only if the functions $\mathrm{g}_{1}$ and $M_{2}$ are in involution

$$
\left[\mathrm{g}_{1}(q, p), M_{2}(q, p)\right]=0 \text { for all }(q, p) \in D \text {. }
$$

Proof. By the identity (3.2) under $M_{1}=0$, it follows that the derivative of the Poisson bracket (1.2) by virtue of the Hamiltonian system (1.1)

$$
\mathfrak{G}\left[\mathrm{g}_{1}(q, p), \mathrm{g}_{2}(q, p)\right]=M_{2}(q, p)\left[\mathrm{g}_{1}(q, p), \mathrm{g}_{2}(q, p)\right]+\mathrm{g}_{2}(q, p)\left[\mathrm{g}_{1}(q, p), M_{2}(q, p)\right] .
$$

Therefore, the Poisson bracket (1.2) is a partial integral with cofactor $M_{2}$ of the Hamiltonian system (1.1) if and only if the identity (3.5) holds.

Theorem 5. Under the conditions of Proposition 2, we see that the Poisson bracket (1.2) is a first integral of the Hamiltonian system (1.1) if and only if the following identity holds:

$$
M_{2}(q, p)\left[\mathrm{g}_{1}(q, p), \mathrm{g}_{2}(q, p)\right]+\mathrm{g}_{2}(q, p)\left[\mathrm{g}_{1}(q, p), M_{2}(q, p)\right]=0 \text { for all }(q, p) \in D .
$$

Proof. The proof follows from the identity (3.6).

Theorem 6. If the functions $\mathrm{g}_{1} \in \mathrm{I}(\mathfrak{G}),\left(\mathrm{g}_{2}, M_{2}\right) \in \mathrm{J}(\mathfrak{G}), \mathrm{g}_{k} \in C^{2}(D), k=1,2$, such that the following identity holds:

$$
\left[\mathrm{g}_{1}(q, p), M_{2}(q, p)\right]=\alpha M_{2}(q, p) \text { for all }(q, p) \in D, \quad \alpha \in \mathbb{R},
$$

then a first integral of the Hamiltonian system (1.1) is the function

$$
\mathrm{g}(q, p)=\mathrm{g}_{2}^{\alpha}(q, p) \exp \left(-\frac{\left[\mathrm{g}_{1}(q, p), \mathrm{g}_{2}(q, p)\right]}{\mathrm{g}_{2}(q, p)}\right) \text { for all }(q, p) \in D_{0},
$$

where $D_{0}$ is a domain from the set $\left\{(q, p) \in D: \mathrm{g}_{2}(q, p) \neq 0\right\}$.

Proof. Using the identity (3.6) under (3.8) and $\left(\mathrm{g}_{2}, M_{2}\right) \in \mathrm{J}(\mathfrak{G})$, we obtain the derivative of the function (3.9) by virtue of the Hamiltonian system (1.1) on the domain $D_{0}$

$$
\begin{aligned}
\mathfrak{G} \mathrm{g}(q, p) & =\alpha \mathrm{g}_{2}^{\alpha-1}(q, p) \exp \left(-\frac{\left[\mathrm{g}_{1}(q, p), \mathrm{g}_{2}(q, p)\right]}{\mathrm{g}_{2}(q, p)}\right) \mathfrak{G} \mathrm{g}_{2}(q, p)- \\
& -\mathrm{g}(q, p) \cdot \frac{\mathrm{g}_{2}(q, p) \mathfrak{G}\left[\mathrm{g}_{1}(q, p), \mathrm{g}_{2}(q, p)\right]-\left[\mathrm{g}_{1}(q, p), \mathrm{g}_{2}(q, p)\right] \mathfrak{G}_{2}(q, p)}{\mathrm{g}_{2}^{2}(q, p)}=0 .
\end{aligned}
$$

For example, the system (1.1) with the Hamiltonian

$$
H(q, p)=q_{1} p_{1}-q_{2} p_{2}-a q_{1}^{2}+b q_{2}^{2} \quad \text { for all }(q, p) \in \mathbb{R}^{4}, \quad a, b \in \mathbb{R},
$$


has the first integral $\mathrm{g}_{1}(q, p)=\frac{p_{1}-a q_{1}}{q_{2}}$ on the domain $D \subset\left\{(q, p): q_{2} \neq 0\right\}$ and the partial integral $\mathrm{g}_{2}(q, p)=p_{2}-b q_{2}$ with the cofactor $M_{2}(q, p)=1$ for all $(q, p) \in \mathbb{R}^{4}$. Using Theorem 6 under $\alpha=0$, we can build the additional first integral of the Hamiltonian system (3.10)

$$
\mathrm{g}(q, p)=-\frac{\left[\mathrm{g}_{1}(q, p), \mathrm{g}_{2}(q, p)\right]}{\mathrm{g}_{2}(q, p)}=-\frac{\left[\frac{p_{1}-a q_{1}}{q_{2}}, p_{2}-b q_{2}\right]}{p_{2}-b q_{2}}=\frac{p_{1}-a q_{1}}{q_{2}^{2}\left(p_{2}-b q_{2}\right)} \text { for all }(q, p) \in D_{0},
$$

where the domain $D_{0} \subset\left\{(q, p): q_{2}\left(p_{2}-b q_{2}\right) \neq 0\right\}$.

\section{Application}

In this section, we extend the results obtained in Sections 2 and 3 for the Hamiltonian system (1.1) on an $n$th order ordinary differential system

$$
\frac{d q_{i}}{d t}=X_{i}(q), \quad i=1, \ldots, n,
$$

where the functions $X_{i}$ are continuously differentiable on the domain $Q \subset \mathbb{R}^{n}$.

Using the Liouville method [20, pp. 429-430], we can build the following augmented Hamiltonian system for the differential system (4.1)

$$
\frac{d q_{i}}{d t}=\partial_{p_{i}} H(q, p), \quad i=1, \ldots, n, \quad \frac{d p_{i}}{d t}=-\partial_{q_{i}} H(q, p), \quad i=1, \ldots, n,
$$

with Hamiltonian $H(q, p)=\sum_{i=1}^{n} X_{i}(q) p_{i}$ for all $(q, p) \in Q \times \mathbb{R}^{n}$.

The Hamiltonian system (4.2) is such that the first $n$ equations are the system (4.1) and the second $n$ equations are the auxiliary system for defining redundant variables $p_{1}, \ldots, p_{n}$.

Moreover, the auxiliary differential system has the form

$$
\frac{d p_{i}}{d t}=-\sum_{j=1}^{n} \partial_{q_{i}} X_{j}(q) p_{j}, \quad i=1, \ldots, n
$$

The system (4.1) and the augmented Hamiltonian system (4.2) induce, respectively, the first-order linear differential operators

$$
\mathfrak{X}(q)=\sum_{i=1}^{n} X_{i}(q) \partial_{q_{i}} \text { for all } q \in Q
$$

and

$$
\begin{aligned}
\mathfrak{G}(q, p) & =\sum_{i=1}^{n} X_{i}(q) \partial_{q_{i}}-\sum_{i=1}^{n} \sum_{j=1}^{n} \partial_{q_{i}} X_{j}(q) p_{j} \partial_{p_{i}}= \\
& =\mathfrak{X}(q)-\sum_{i=1}^{n} \sum_{j=1}^{n} \partial_{q_{i}} X_{j}(q) p_{j} \partial_{p_{i}} \quad \text { for all }(q, p) \in Q \times \mathbb{R}^{n}
\end{aligned}
$$




\subsection{Analytical relations between systems (4.1) and (4.2)}

Lemma 1 ([21, p. 111]). Suppose $\mathrm{g} \in C^{2}(Q)$ is a first integral on the domain $Q$ of the differential system (4.1). Then the functions

$$
p_{i}(q)=\partial_{q_{i}} \mathrm{~g}(q) \text { for all } q \in Q, \quad i=1, \ldots, n,
$$

are a partial solution to the auxiliary differential system (4.3).

Lemma 2. A continuously differentiable function

$$
\widetilde{\mathrm{g}}(q, p)=\mathrm{g}(q) \text { for all }(q, p) \in Q \times \mathbb{R}^{n}
$$

defines an n-cylindrical integral manifold of the augmented Hamiltonian differential system (4.2) if and only if the scalar function

$$
\mathrm{g}(q) \text { for all } q \in Q
$$

defines an integral manifold of the ordinary differential system (4.1).

Proof. Necessity. If the function (4.5) defines an $n$-cylindrical integral manifold of the augmented Hamiltonian system (4.2), then (by the definition of integral manifold) we have the identity

$$
\mathfrak{G} \widetilde{\mathrm{g}}(q, p)=\Phi(q, p) \text { for all }(q, p) \in Q \times \mathbb{R}^{n} .
$$

Now, taking into account $\mathfrak{G} \widetilde{\mathrm{g}}(q, p)=\mathfrak{X} \mathrm{g}(q)$, we obtain the identity

$$
\mathfrak{X} \mathrm{g}(q)=\Phi(q, p) \text { for all }(q, p) \in Q \times \mathbb{R}^{n},
$$

where the function $\left.\Phi(q, p)\right|_{\mathrm{g}(q)=0}=0$ for all $(q, p) \in Q \times \mathbb{R}^{n}$.

Since the left-hand side of identity (4.7) does not depend on the variable $p$, we see that the function $\Phi$ from identity (4.7) is $n$-cylindrical with respect to the variable $p$, i.e.,

$$
\Phi:(q, p) \rightarrow \Phi(q) \text { for all }(q, p) \in Q \times \mathbb{R}^{n} .
$$

This implies that the identity (4.7) has the form

$$
\mathfrak{X} \mathrm{g}(q)=\Phi(q) \text { for all } q \in Q,
$$

where the function $\left.\Phi(q)\right|_{\mathrm{g}(q)=0}=0$ for all $q \in Q$.

Thus, the function (4.6) defines an integral manifold of the differential system (4.1).

Sufficiency follows from $(4.8)$ and $\mathfrak{X} g(q)=\mathfrak{G} \widetilde{\mathrm{g}}(q, p)$ for all $(q, p) \in Q \times \mathbb{R}^{n}$.

As a consequence of Lemma 2, we obtain the following

Lemma $3([21$, p. $112 ; 18])$. A continuously differentiable function $(4.5)$ is an $n$-cylindrical first integral of the augmented Hamiltonian differential system (4.2) if and only if the function (4.6) is a first integral of the differential system (4.1).

In the case of the polynomial differential system (4.1), from Lemma 2, we get the following

Lemma 4. A continuously differentiable function (4.5) is an n-cylindrical partial integral of the augmented Hamiltonian differential system (4.2) if and only if the function (4.6) is a partial integral of the polynomial differential system (4.1). 


\subsection{Integral manifolds and partial integrals}

Using integrals and integral manifolds of the Hamiltonian system (4.2), we can build (by Theorems 7-11 and Corollaries 2-5) integral characteristics for system (4.1).

Theorem 7. Suppose $\mathrm{g} \in \mathrm{I}(\mathfrak{X}),\left(\mathrm{g}_{1}, \Phi_{1}\right) \in \mathrm{M}(\mathfrak{G})$. Then, if the function

$$
\widetilde{\mathrm{g}}_{1}(q)=\mathrm{g}_{1}(q, p)_{\mid p=\partial_{q} \mathrm{~g}(q)} \text { for all } q \in Q
$$

defines a manifold, then this manifold is an integral manifold of the differential system (4.1).

Proof. Since the function (4.4) is a partial solution (Lemma 1) of system (4.3), we see that the differential of the function (4.9) by virtue of the system (4.1)

$$
\begin{gathered}
d \widetilde{\mathrm{g}}_{1}(q)_{\mid(4.1)}=\left.\sum_{i=1}^{n} \partial_{q_{i}} \mathrm{~g}_{1}(q, p)\right|_{p=\partial_{q} \mathrm{~g}(q)} X_{i}(q) d t+\sum_{i=1}^{n} \partial_{p_{i}} \mathrm{~g}_{1}(q, p)_{\mid p=\partial_{q} \mathrm{~g}(q)}\left(-\sum_{j=1}^{n} \partial_{q_{i}} X_{j}(q) p_{j}\right) d t= \\
=\left(\sum_{i=1}^{n} X_{i}(q) \partial_{q_{i}} \mathrm{~g}_{1}(q, p)-\sum_{i=1}^{n} \sum_{j=1}^{n} \partial_{q_{i}} X_{j}(q) p_{j} \partial_{p_{i}} \mathrm{~g}_{1}(q, p)\right)_{\mid p=\partial_{q} \mathrm{~g}(q)} d t=\mathfrak{G} \mathrm{g}_{1}(q, p)_{{ }_{p=\partial_{q} \mathrm{~g}(q)}} d t .
\end{gathered}
$$

Now from $\left(\mathrm{g}_{1}, \Phi_{1}\right) \in \mathrm{M}(\mathfrak{G})$ it follows that

$$
d \widetilde{\mathrm{g}}_{1}(q)_{\mid(4.1)}=\Phi_{1}(q, p)_{\left.\right|_{p=\partial_{q} \mathrm{~g}(q)}} d t=\widetilde{\Phi}_{1}(q) d t,
$$

where the function $\widetilde{\Phi}_{1}(q)_{\mid \widetilde{\mathrm{g}}_{1}(q)=0}=\left(\Phi_{1}(q, p)_{\left.\right|_{\mathrm{g}_{1}(q, p)=0}}\right)_{\mid p=\partial_{q} \mathrm{~g}(q)}=0$.

Thus, the function (4.9) is an integral manifold of system (4.1), i.e., $\left(\widetilde{\mathrm{g}}_{1}, \widetilde{\Phi}_{1}\right) \in \mathrm{M}(\mathfrak{X})$.

Corollary 2. Let $\mathrm{g} \in \mathrm{I}(\mathfrak{X}),\left(\mathrm{g}_{1}, M_{1}\right) \in \mathrm{J}(\mathfrak{G})$. Then, if the function (4.9) defines a manifold, then this manifold is an integral manifold of the polynomial system (4.1) and

$$
\widetilde{\Phi}_{1}(q)=\mathrm{g}_{1}(q, p) M_{1}(q, p)_{\left.\right|_{p=\partial_{q} \mathrm{~g}(q)}} \text { for all } q \in Q .
$$

Moreover, if $\widetilde{M}_{1}(q)=M_{1}(q, p)_{\mid p=\partial_{q} \mathrm{~g}(q)}$ for all $q \in Q$ is a polynomial in the variable $q$, then the function (4.9) is a partial integral of the polynomial system (4.1), i.e., $\left(\widetilde{\mathrm{g}}_{1}, \widetilde{M}_{1}\right) \in \mathrm{J}(\mathfrak{X})$.

Corollary 3 ([21, p. 112]). If $\mathrm{g} \in \mathrm{I}(\mathfrak{X})$ and $\mathrm{g}_{1} \in \mathrm{I}(\mathfrak{G})$, then the function (4.9) is a first integral of the differential system (4.1).

The analogue of Theorem 2 for the ordinary differential system (4.1) is the following

Theorem 8. Suppose $\mathrm{g} \in \mathrm{I}(\mathfrak{X})$ and $\left(\mathrm{g}_{k}, \Phi_{k}\right) \in \mathrm{M}(\mathfrak{G}), \mathrm{g}_{k} \in C^{2}(D), k=1,2$, such that the identity (2.1) holds on the domain $D=Q \times P, P \subset \mathbb{R}^{n}$. Then the Poisson bracket

$$
\widetilde{\mathrm{g}}_{12}(q)=\left[\mathrm{g}_{1}(q, p), \mathrm{g}_{2}(q, p)\right]_{\left.\right|_{p=\partial_{q} \mathrm{~g}(q)}} \text { for all } q \in Q
$$

is a first integral of the differential system (4.1).

Proof. Let $\left(\mathrm{g}_{k}, \Phi_{k}\right) \in \mathrm{M}(\mathfrak{G})$. By Theorem 2, the function (1.2) is a first integral of the Hamiltonian system (4.2).

Using the first integrals $\mathrm{g} \in \mathrm{I}(\mathfrak{X})$ and $\mathrm{g}_{12} \in \mathrm{I}(\mathfrak{G})$, by Corollary 3, we can build the first integral (4.10) of the ordinary differential system (4.1). 
REMARK 1. Suppose $g_{1}$ and $g_{2}$ are infinitely continuously differentiable functions with respect to the variable $q$. Then, under the conditions of Theorem 8 , we find that the functions

$$
\widetilde{\mathrm{g}}_{12, k}(q)=\left[\mathrm{g}_{1}(q, p), \mathrm{g}_{2}(q, p)\right]_{p=\partial_{q} \widetilde{\mathrm{g}}_{12, k-1}(q)} \text { for all } q \in Q, \quad k=1,2, \ldots, \quad\left(\widetilde{\mathrm{g}}_{12,0}=\mathrm{g}\right),
$$

are also first integrals of the ordinary differential systems (4.1).

REMARK 2. If the functions $\mathrm{g}_{0 \xi} \in \mathrm{I}(\mathfrak{X}), \xi=1, \ldots, m$, are functionally independent first integrals on the domain $Q$ of system (4.1), then the functions (by Theorem 8)

$$
\widetilde{\mathrm{g}}_{12, \xi}(q)=\left[\mathrm{g}_{1}(q, p), \mathrm{g}_{2}(q, p)\right]_{p=\partial_{q} \mathrm{~g}_{0 \xi}(q)} \text { for all } q \in Q, \quad \xi=1, \ldots, m,
$$

are also first integrals of the ordinary differential systems (4.1).

If one of the integral manifolds is $n$-cylindrical with respect to the variable $p$, then from Theorem 8, Lemma 2, and the definition of Poisson bracket we have

Corollary 4. Suppose $\mathrm{g} \in \mathrm{I}(\mathfrak{X})$, the integral manifolds $\left(\mathrm{g}_{1}, \Phi_{1}\right) \in \mathrm{M}(\mathfrak{X}), \mathrm{g}_{1} \in C^{2}(Q)$, and $\left(\mathrm{g}_{2}, \Phi_{2}\right) \in \mathrm{M}(\mathfrak{G}), \mathrm{g}_{2} \in C^{2}(D)$, such that the following identity holds:

$$
\sum_{i=1}^{n} \partial_{q_{i}} \mathrm{~g}_{1}(q) \partial_{p_{i}} \Phi_{2}(q, p)=-\sum_{i=1}^{n} \partial_{q_{i}} \Phi_{1}(q) \partial_{p_{i}} \mathrm{~g}_{2}(q, p) \quad \text { for all }(q, p) \in D=Q \times P \subset \mathbb{R}^{2 n} \text {. }
$$

Then a first integral of the differential system (4.1) is the function

$$
\widetilde{\mathrm{g}}_{12}(q)=\sum_{i=1}^{n} \partial_{q_{i}} \mathrm{~g}_{1}(q) \partial_{p_{i}} \mathrm{~g}_{2}(q, p)_{\mid p=\partial_{q} \mathrm{~g}(q)} \text { for all } q \in Q
$$

From the Poisson theorem (Theorem 1), Lemma 3, and Corollary 3, we get the generalized Poisson theorem [21, p. 112; 18] of building first integrals for the differential system (4.1).

Corollary 5 (the generalized Poisson theorem). If $\mathrm{g}_{1} \in \mathrm{I}(\mathfrak{X}), \mathrm{g}_{1} \in C^{2}(Q)$, and $\mathrm{g}_{2} \in \mathrm{I}(\mathfrak{G}), \mathrm{g}_{2} \in C^{2}(D)$, then a first integral of system (4.1) is the Poisson bracket

$$
\mathrm{g}_{12}^{*}(q)=\left[\mathrm{g}_{1}(q), \mathrm{g}_{2}(q, p)\right]_{p=\partial_{q} \mathrm{~g}_{1}(q)} \text { for all } q \in Q
$$

Using the main statements (Theorems 4, 5, and 6) of Section 3 and Corollary 3, we obtain the approach (Theorems 9, 10, and 11) to building an additional first integral of the polynomial differential system (4.1) by the known first integral of the polynomial differential system (4.1) and the known partial integrals of the Hamiltonian system (4.2).

Theorem 9. If $\mathrm{g} \in \mathrm{I}(\mathfrak{X})$ and $\left(\mathrm{g}_{k}, M_{k}\right) \in \mathrm{J}(\mathfrak{G}), \mathrm{g}_{k} \in C^{2}(D), k=1,2$, such that the identities (3.3) and (3.4) are true, then the Poisson bracket (4.10) is a first integral of the polynomial differential system (4.1).

Theorem 10. Suppose $\mathrm{g}_{1} \in \mathrm{I}(\mathfrak{X})$ and $\left(\mathrm{g}_{2}, M_{2}\right) \in \mathrm{J}(\mathfrak{G}), \mathrm{g}_{k} \in C^{2}(D), k=1,2$, such that the identity (3.7) holds under $\mathrm{g}_{1}(q, p)=\mathrm{g}_{1}(q)$ for all $(q, p) \in D$. Then the Poisson bracket (4.10) is a first integral of the polynomial differential system (4.1).

Theorem 11. Suppose $\mathrm{g}_{1} \in \mathrm{I}(\mathfrak{X})$ and $\left(\mathrm{g}_{2}, M_{2}\right) \in \mathrm{J}(\mathfrak{G}), \mathrm{g}_{k} \in C^{2}(D), k=1,2$, such that the identity (3.8) holds under $\mathrm{g}_{1}(q, p)=\mathrm{g}_{1}(q)$ for all $(q, p) \in D$. Then the function

$$
\widetilde{\mathrm{g}}_{12}(q)=\mathrm{g}_{2}^{\alpha}(q, p) \exp \left(-\frac{\left[\mathrm{g}_{1}(q), \mathrm{g}_{2}(q, p)\right]}{\mathrm{g}_{2}(q, p)}\right)_{\mid p=\partial_{q} \mathrm{~g}_{1}(q)} \text { for all } q \in Q
$$

is a first integral of the polynomial differential system (4.1). 
The author thanks Prof. Victor N. Gorbuzov for useful discussions. Also, the author would like to thank the anonymous referees for careful reading of the manuscript and suggesting a number of changes that helped to improve the paper.

\section{References}

[1] Jacobi, C. G. J., Jacobi's Lectures on Dynamics, A. Clebsch (Ed.), 2nd ed., New Delhi: Hindustan Book Agency, 2009.

[2] Arnol'd, V.I., Mathematical Methods of Classical Mechanics, 2nd ed., Grad. Texts in Math., vol.60, New York: Springer, 1989.

[3] Gantmacher, F. R., Lectures in Analytical Mechanics, Moscow: Mir, 1975.

[4] Gorbuzov, V. N., Integrals of Differential Systems, Grodno: GrGU, 2006 (Russian).

[5] Darboux, G., Mémoire sur les équations différentielles algébriques du premier ordre et du premier degré, Bulletin des Sciences Mathématiques et Astronomiques, Sér. 2, 1878, vol. 2, no. 1, pp.60-96, 123-144, 151-200.

[6] Poincaré, H., Sur l'intégration algébrique des équations différentielles du premier ordre et du premier degré: 1, Rend. Circ. Mat. Palermo, 1891, vol.5, pp. 161-191;

Poincaré, H., Sur l'intégration algébrique des équations différentielles du premier ordre et du premier degré: 2, Rend. Circ. Mat. Palermo, 1897, vol. 11, pp. 193-239.

[7] Hilbert's Problems, P.S. Alexandrov (Ed.), Moscow: Nauka, 1969 (Russian).

[8] Kozlov, V. V., Symmetries, Topology and Resonances in Hamiltonian Mechanics, Ergeb. Math. Grenzgeb. (3), vol.31, Berlin: Springer, 1996.

[9] Goriely, A., Integrability and Nonintegrability of Dynamical Systems, Adv. Ser. Nonlinear Dynam., vol. 19, River Edge, N.J.: World Sci., 2001.

[10] Borisov, A. V. and Mamaev, I. S., Modern Methods of the Theory of Integrable Systems, Moscow: R\&C Dynamics, ICS, 2003 (Russian).

[11] Llibre, J., Integrability of Polynomial Differential Systems, in Handbook of Differential Equations: Ordinary differential equations: Vol. 1, A. Cañada, P. Drábek, A. Fonda (Eds.), Amsterdam: Elsevier/North-Holland, 2004, pp. 437-532.

[12] Pranevich, A.F., R-differentiable Integrals for Systems of Equations in Total Differentials, Saarbrücken: Lambert, 2011 (Russian).

[13] Zhang, X., Integrability of Dynamical Systems: Algebra and Analysis, Dev. Math., vol. 47, Singapore: Springer, 2017.

[14] Maciejewski, A. J. and Przybylska, M., Darboux Polynomials and First Integrals of Natural Polynomial Hamiltonian Systems, Phys. Lett. A, 2004, vol. 326, nos. 3-4, pp. 219-226.

[15] Nakagawa, K., Maciejewski, A. J., and Przybylska, M., New Integrable Hamiltonian Systems with First Integrals Quartic in Momenta, Phys. Lett. A, 2005, vol. 343, nos. 1-3, pp. 171-173.

[16] Llibre, J., Stoica, Ch., and Valls, C., Polynomial and Rational Integrability of Polynomial Hamiltonian Systems, Electron. J. Differential Equations, 2012, No. 108, 6 pp.

[17] Gorbuzov, V.N. and Pranevich, A.F., First Integrals of Ordinary Linear Differential Systems, arXiv:1201.4141 (2012).

[18] Pranevich, A.F., Poisson Theorem of Building Autonomous Integrals for Autonomous Systems of Total Differential Equations, Problemy Fiziki, Matematiki i Tekhniki, 2016, no. 3(28), pp. 52-57 (Russian).

[19] Kozlov, V. V., Linear Hamiltonian Systems: Quadratic Integrals, Singular Subspaces and Stability, Regul. Chaotic Dyn., 2018, vol. 23, no. 1, pp. 26-46.

[20] Appell, P., Traité de mécanique rationnelle: Vol. 2, Paris: Gauthier-Villars, 1953.

[21] Shul'gin, M.F., On Some Differential Equations of Analytical Dynamics and Their Integration, Tashkent: SAGU, 1958 (Russian). 